\title{
Fragility and the dynamic crossover in lubricants
}

\author{
S Bair ${ }^{1 *}$, C M Roland ${ }^{2}$, and R Casalini' ${ }^{2,3}$ \\ ${ }^{1}$ George W Woodruff School of Mechanical Engineering, Georgia Institute of Technology, Center for High Pressure \\ Rheology, Atlanta, Georgia, USA \\ ${ }^{2}$ Naval Research Laboratory, Chemistry Division, Washington, District of Columbia, USA \\ ${ }^{3}$ Chemistry Department, George Mason University, Fairfax, Virginia, USA
}

The manuscript was received on 31 January 2007 and was accepted after revision for publication on 26 July 2007.

DOI: 10.1243/13506501JET278

\begin{abstract}
The property of fragility in glass forming liquids is introduced to elastohydrodynamic lubrication (EHL). Using viscosity measurements for three liquids that have been the subject of traction studies the fragility of liquids is shown to be important to EHL traction and the property that most influences the representative (Eyring) stress. The derivative Stickel analysis is then carried out for lubricants. Using viscosity measurements, the dynamic crossover is detected for the first time in lubricants. The viscosity at the crossover is either constant or varies slowly with temperature and pressure and the crossover pressure varies with temperature in a manner similar to the glass transition pressure. The free-volume model fails to predict the occurrence of the dynamic crossover.
\end{abstract}

Keywords: elastohydrodynamic lubrication, fragility, traction, pressure-viscosity behaviour, Eyring stress

\section{INTRODUCTION}

Elastohydrodynamic lubrication (EHL) refers to the piezoviscous liquid films separating high elastic modulus machine elements at the non-conformal contacts that occur in everyday machines such as gears and rolling element bearings. The measurable operating parameters of practical importance for a contact are the film thickness and the traction (friction). Film thickness provides durability for the surfaces and traction is responsible for mechanical energy dissipation. Film thickness is essentially established in a low-pressure region at the inlet side of the contact, whereas traction, for the most part, is generated by the shearing of the film in the high-pressure, Hertz region of contact. For low-molecular weight liquids, the rheological response within the film-forming inlet is Newtonian and the film thickness may be calculated with excellent accuracy [1] from measurements

*Corresponding author: George W Woodruff School of Mechanical Engineering, Georgia Institute of Technology, Center for High Pressure Rheology, Atlanta, GA 30332-0405, USA. email: scott.bair@me.gatech.edu of the liquid low-shear viscosity and compressibility over the range of pressures existing within the contact. The pressure within the film-forming inlet is quite low, reaching only to about $p=3 / \alpha^{*}(100-400 \mathrm{MPa})$, where the film-forming pressure-viscosity coefficient may be given by

$$
\alpha^{*}(T)=\left[\int_{0}^{\infty} \frac{\mu(p=0)}{\mu(p)} \mathrm{d} p\right]^{-1}
$$

The strength of the piezoviscous response within the inlet zone may be approximately quantified by equation (1) for determining the Newtonian film thickness. Success in understanding the mechanism of film generation for Newtonian liquids came early through numerical simulations solving the Reynolds equation $[2,3]$ in combination with the elastic response of the rollers, the 'full EHL solution'.

Traction, on the other hand, has not easily yielded to numerical simulation of the full EHL problem. The impediment is the large value of the local pressureviscosity coefficient

$$
\alpha(T, p)=\frac{\partial(\ln \mu)}{\partial p}
$$


at the pressures prevailing in the Hertz region. First, the usual algorithms for the solution of the combined Reynolds and elasticity equations become unstable [4] for realistically large values of $\alpha$. Second, as pointed out by Brix [5] some fifty-five years ago, the Reynolds equation was derived under the assumption of constant viscosity. A piezoviscous liquid will result in significant spatial viscosity derivatives that invalidate this basic equation of hydrodynamic lubrication when the product of pressure-viscosity coefficient and shear stress, $\alpha \tau$, approaches unity [6, 7]. Further hampering progress in traction modelling, in contrast to film thickness modelling, is the apparent lack, except at very low pressures, of a measurable traction which can be attributed to Newtonian response alone.

Two approaches have therefore evolved for the study of traction. In the first, the relationship between the average shear stress in a traction measurement and the slide-to roll ratio, $\Sigma$, of the contacting bodies is considered to have the same functional form as a rheological constitutive law. A sinh-law is assumed for this purpose as a reasonable description of a traction curve at intermediate contact pressures $[\mathbf{8}, \mathbf{9}]$

$$
\tau_{\mathrm{ave}}=\tau_{\mathrm{E}} \sinh ^{-1}\left(C_{\mathrm{E}} \Sigma\right)
$$

Here, $C_{\mathrm{E}}$ is a constant for a given load, temperature, and rolling velocity and $\tau_{E}$ is a representative stress (also known as Eyring stress), equal to the slope of a logarithmic traction gradient. The shear stress, averaged over the contact area, is $\tau_{\text {ave }}$.

In the second approach, viscometers are employed to measure the generalized viscosity, $\eta(T, p, \tau)$, over an experimentally convenient range of temperature, pressure and shear stress. Models are fitted to these data and the calculated stress integrated over the Hertz region to arrive at the traction. This technique offers the advantage of relating traction to measurable properties which, in the case of high-molecular weight liquids, are also useful for the prediction of film thickness. The disadvantage is that these models could not, until very recently, be incorporated in the usual full EHL numerical solutions. Several laboratories have made progress in generating full numerical solutions $[1,10,11]$ using realistic pressure and shear dependence of viscosity to yield excellent agreement with measured film thickness and traction. The EHL field is on the verge of a significant move toward quantitative calculations which would provide insight, for example, into what constitutes a good EHL lubricant. Such calculations require an accurate description of the pressure dependence of viscosity rather than the usual Roelands extrapolation. The most obvious choice for accurate temperature and pressure dependence has been the free-volume model.

The current paper introduces the concept of fragility to EHL and establishes a relationship between pressure fragility and the representative stress in EHL traction. Fragile liquids sometimes display a dynamic crossover. The first observations of a dynamic crossover are reported in EHL lubricants and it is demonstrated that the free-volume approach fails when the crossover is encountered. The dynamic crossover, therefore, represents a limitation to EHL calculations employing free-volume. Henceforth the term, viscosity, will be used to indicate only the limiting-low-shear stress value.

\section{FRAGILITY}

The term 'fragility' is used to describe differences in the temperature dependences of supercooled, glassforming liquids [12-15]. Fragile liquids experience greater changes in their properties (are more nonArrhenius) as the glass transition is approached by cooling than do strong liquids. Fragile behaviour suggests that the short range order of the glass is more rapidly disrupted by an increase in temperature, although the terminology per se does not rely on the correctness of this interpretation.

Ordinarily, fragility is illustrated with a plot of the logarithm of viscosity, $\mu$, against reciprocal absolute temperature normalized by the glass transition temperature, $T_{\mathrm{g}} / T$. See Fig. 1 where it has been assumed that the viscosity at the glass transition, $\mu_{\mathrm{g}}=10^{10} \mathrm{~Pa} \mathrm{~s}$. An Arrhenius response corresponds to an ascending straight line, indicating the strongest (or least fragile) behaviour, with the viscosity following the Andrade law [16].

$$
\mu=\mu_{\infty} \exp \left[\frac{E_{\mathrm{a}}}{R_{\mathrm{g}} T}\right]
$$

This is the behaviour of a strictly thermally-activated liquid. A more fragile response appears in this

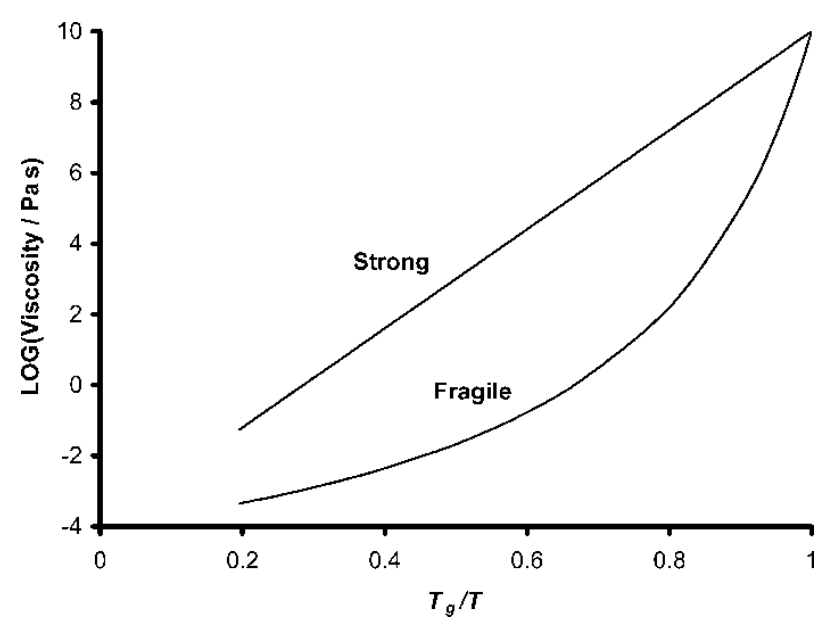

Fig. $1 T_{\mathrm{g}}$-normalized Arrhenius plot illustrating the concept of fragility 
plot as an upward convex curve, which can be described by the Vogel, Tammann, and Fulcher (VTF) equation $[14,15]$.

$$
\mu=\mu_{\infty} \exp \left[\frac{D_{\mathrm{F}} T_{\infty}}{T-T_{\infty}}\right]
$$

Here, $D_{\mathrm{F}}$ is a constant and $T_{\infty}$, the Vogel temperature, is the temperature at which the viscosity diverges. The more fragile liquid exhibits greater departure from Arrhenius behaviour.

The pressure counterpart of the VTF equation is the Johari and Whalley [17] (J\&W) equation

$$
\mu=\mu_{\infty} \exp \left[\frac{C_{\mathrm{F}} p_{\infty}}{p_{\infty}-p}\right]
$$

where $C_{\mathrm{F}}$ is referred to [16] as the pressure-fragility parameter. The equivalent form of relations (5) and (6) suggests that there should be a pressure counterpart to the temperature fragility plot as well. If the reciprocal of temperature is substituted for the pressure in equation (6) with the appropriate fragility parameter, the resulting form is the VTF equation (5). The proposed pressure embodiment of the fragility plot is shown in Fig. 2(a), where the logarithm of viscosity is plotted against the pressure scaled by the glass transition pressure, $p / p_{\mathrm{g}}$, and once again $\mu_{\mathrm{g}}=10^{10} \mathrm{~Pa}$ s. The two liquids shown in Fig. 2, are an $8 \mathrm{cSt}$ commercial grade of polyalphaolefin (PAO) and the model lubricant [8], dibenzylethylbenzene (DBEB) with properties listed in Table 1. Using the classification method of Fig. 1, the DBEB is more fragile than the PAO. The scaling of pressure by the glass transition pressure, employed in Fig. 2(a), somewhat obscures the true difference in pressure dependence of the viscosity of the two liquids since the glass transition pressure of the DBEB is about one-fourth that of the PAO. An alternative representation is shown in Fig. 2(b).

The metric of temperature fragility is [18]

$$
m_{\mathrm{T}}=\left[\frac{\partial \ln \mu}{\partial\left(T_{\mathrm{g}} / T\right)}\right]_{T=T_{\mathrm{g}}}
$$

Here, the natural logarithm is substituted for the common logarithm so that a simple relation with the temperature-viscosity coefficient, $\beta=-\partial \ln \mu / \partial T$, may be obtained

$$
m_{\mathrm{T}}=[\beta T]_{T=T_{\mathrm{g}}}
$$

Similarly, Paluch et al. [19] have defined a pressurefragility index as

$$
m_{\mathrm{p}}^{*}=\left[\frac{\partial \ln \mu}{\partial\left(p / p_{\mathrm{g}}\right)}\right]_{p=p_{\mathrm{g}}}=[\alpha p]_{p=p_{\mathrm{g}}}
$$
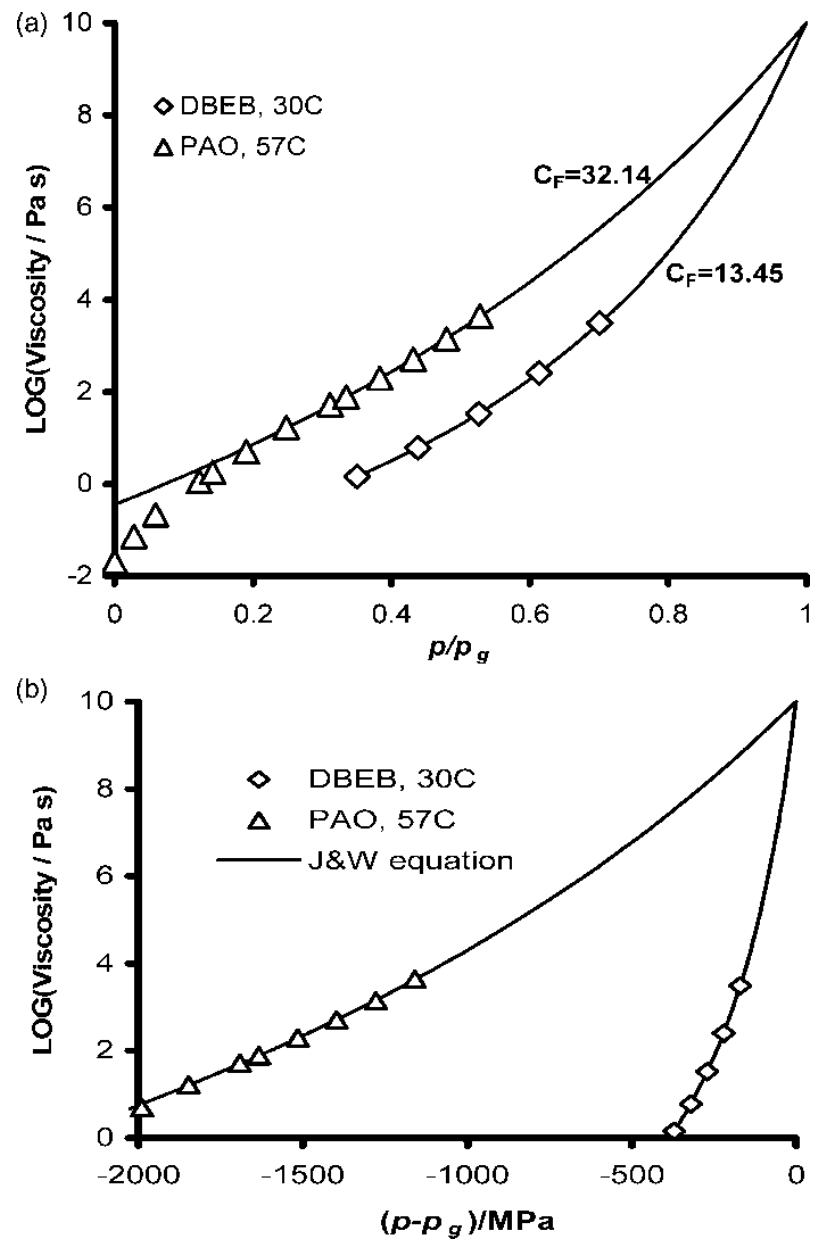

Fig. 2 (a) The pressure counterpart of the fragility plot in Fig. 1 for two liquids that have been employed for traction measurements, (b) an alternative representation of the pressure dependence. The stronger liquid, PAO, displays a large value of representative stress while the more fragile liquid, DBEB, displays an exceptionally low representative stress. The curves are the J\&W equation (6)

Although this definition is dimensionless like the temperature version, it is undefined for cases where $p_{\mathrm{g}} \rightarrow 0$. An earlier definition by Paluch et al. [20] does not scale the pressure by $p_{\mathrm{g}}$ and will be more useful for

Table 1 Properties of the liquids shown in Fig. 2 and a mineral oil, LVI260

\begin{tabular}{llll}
\hline & PAO & DBEB & LVI260 \\
\hline$T(\mathrm{~K})$ & 330 & 303 & 353 \\
$\mu_{\infty}(\mathrm{Pa} \mathrm{s})$ & $3.89 \times 10^{-15}$ & $3.43 \times 10^{-8}$ & $6.8 \times 10^{-11}$ \\
$C_{\mathrm{F}}$ & 32.14 & 13.45 & 21.7 \\
$p_{\infty}(\mathrm{GPa})$ & 5.73 & 0.857 & 1.61 \\
$p_{\mathrm{g}}^{\mathrm{a}}(\mathrm{GPa})$ & 2.46 & 0.57 & 0.86 \\
$\alpha_{\mathrm{g}}(\mathrm{GPa})^{-1}$ & 17.2 & 145 & 61.7 \\
$\tau_{\mathrm{E}}(\mathrm{MPa})$ & $13.2[\mathbf{2 1}]$ & $0.86[\mathbf{8}]$ & $4[\mathbf{9}]$ \\
\hline
\end{tabular}

${ }^{\mathrm{a}} \mu_{\mathrm{g}}=10^{10} \mathrm{~Pa} \mathrm{~s}$. 
lubricants

$$
m_{\mathrm{p}}=\left[\frac{\partial \ln \mu}{\partial p}\right]_{p=p_{\mathrm{g}}}=\alpha_{\mathrm{g}}
$$

The two liquids shown in Fig. 2, PAO and DBEB, have perhaps the greatest and smallest respective values of representative stress, $\tau_{\mathrm{E}}$, reported in the literature $[\mathbf{9}, \mathbf{2 1}]$ (Table 1 ). The PAO and DBEB also have exceptionally small and large respective values of pressure-fragility indices, $\alpha_{\mathrm{g}}$ (Table 1). This suggests a relationship between $\alpha_{\mathrm{g}}$ and $\tau_{\mathrm{E}}$, fragile liquids having small representative stress and strong liquids having large representative stress. The mineral oil, LVI260, used by Johnson and Tevaarwerk [9], is included in Table 1 as an example of a liquid with intermediate representative stress.

\section{THE STICKEL ANALYSIS}

Stickel et al. [22] have introduced a non-modelspecific derivative analysis of relaxation time and viscosity data. The temperature-Stickel function is

$$
\phi_{\mathrm{T}}=\left[\frac{\partial \ln \mu}{\partial(1 / T)}\right]^{-1 / 2}=\left(T^{2} \beta\right)^{-1 / 2}
$$

A temperature-Stickel plot of $\phi_{\mathrm{T}}$ versus reciprocal temperature results in a horizontal line at $\phi_{\mathrm{T}}=\sqrt{R_{\mathrm{g}} / E_{\mathrm{a}}}$ for an Arrhenius response and a descending straight line of slope equal to $-\sqrt{T_{\infty} / D_{\mathrm{F}}}$ with $y$-intercept of $\sqrt{1 / D_{\mathrm{F}} T_{\infty}}$ for a VTF response. By analogy, the pressureStickel function $[\mathbf{2 3}]$ is

$$
\phi_{\mathrm{p}}=\left[\frac{\partial \ln \mu}{\partial(p)}\right]^{-1 / 2}=\alpha^{-1 / 2}
$$

A pressure-Stickel plot of $\phi_{\mathrm{p}}$ versus pressure results in a horizontal line at $\phi_{\mathrm{p}}=\alpha^{-1 / 2}$ for so-called volume-activated dynamics (known as Barus in EHL) and a descending straight line of slope equal to $-\sqrt{1 / p_{\infty} C_{\mathrm{F}}}$ and a zero intercept of $\sqrt{p_{\infty} / C_{\mathrm{F}}}$ for the J\&W equation (6).

Figure 3 is a pressure-Stickel plot for the mineralbased shell turbine oil, T9. Plotted along with the measured data are three pressure-viscosity functions. The parameter values come from a curve fit in the appropriate range of pressure for each model. These are the Barus (although the Barus equation was linear [24]), $\mu=\mu_{0} \exp (\alpha p)$, with $\mu_{0}=4.29 \mathrm{mPa}$ and $\alpha=14 \mathrm{GPa}^{-1}$, the Roelands [25] isothermal model

$$
\begin{aligned}
\mu= & (63.1 \mu \mathrm{Pa} \mathrm{s}) \exp \left[\ln \left(\frac{\mu_{0}}{63.1 \mu \mathrm{Pas}}\right)\right. \\
& \left.\times\left(1+\frac{p}{0.196 \mathrm{GPa}}\right)^{z}\right]
\end{aligned}
$$

T9 Mineral Oil at $348 \mathrm{~K}$

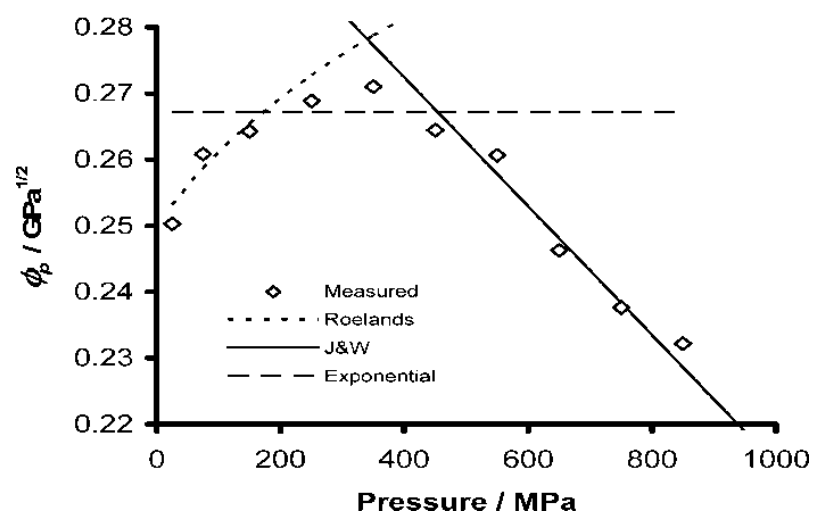

Fig. 3 A pressure-Stickel plot for a commercial turbine oil

with $\mu_{0}=3.39 \mathrm{mPa}$ and $z=0.787$ and the $\mathrm{J} \& \mathrm{~W}$ equation (6) with $\mu_{\infty}=4.4 \times 10^{-14} \mathrm{mPa} \mathrm{s}, C_{\mathrm{F}}=33$, and $p_{\infty}=3.2 \mathrm{GPa}$. Here $\alpha_{\mathrm{g}}=27.4 \mathrm{GPa}^{-1}$.

The Roelands model is the most extensively used pressure-viscosity relation in EHL numerical simulation, although according to Roelands [25] this model is useful only up to the pressure of the inflection, the maximum in the pressure-Stickel plot, and Roelands placed this pressure at 0.1 to $0.5 \mathrm{GPa}$. Measurements show that the inflection may occur at pressures from negative 'tensile' to more than $1 \mathrm{GPa}$ depending on the material and temperature. The J\&W equation (6) can be a useful description of the pressure-dependence of viscosity in the Hertz zone for the calculation of EHL traction in point contact [26] when reproducing the EHL traction behaviour of equation (3). Johnson and Tevaawerk [27] found that for another Shell turbine oil of higher viscosity grade, T33, the sinhlaw of equation (3) provides an accurate description of the average contact shear stress over a three decade range of $10^{-4}<\Sigma<10^{-1}$, with $\tau_{\mathrm{E}} \approx 5 \mathrm{MPa}$. In Fig. 4, the average stress for a circular contact, $\tau_{\mathrm{ave}}=\int_{0}^{1} 2 r \tau \mathrm{d} r$, has been calculated with $p=p_{\mathrm{H}}(1-$ $\left.r^{2}\right)^{1 / 2}$ and the shear stress truncated by the limiting stress-pressure coefficient, $\Lambda=0.07$, using $\tau=$ $\min [\mu \dot{\gamma}, \Lambda p]$. The limit to the shear stress that can be supported by a liquid is believed to result from shear localization [28]. The solid curve in Fig. 4 represents the average stress using the viscosity from the J\&W equation (6) and the above parameters for T9 with the Hertz pressure, $p_{\mathrm{H}}=1.2 \mathrm{GPa}$. The broken curve is the sinh-law (3) with $\tau_{\mathrm{E}}=5 \mathrm{MPa}$. The two curves do not differ by more than $1 \mathrm{MPa}$ over four decades of slide-to-roll ratio. Pressure-fragility, along with a limit to the shear stress is obviously essential to the mechanism of traction at intermediate pressures. 


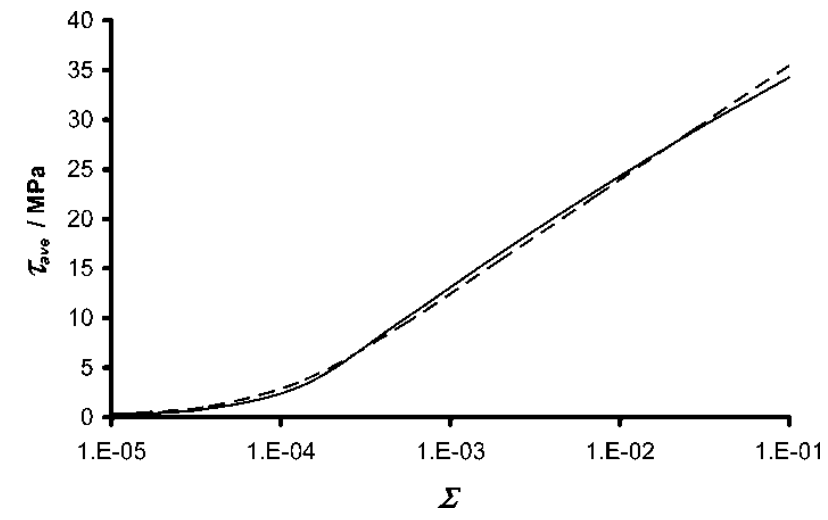

Fig. 4 The solid curve is the shear stress averaged over a circular contact assuming that the pressure dependence of viscosity follows the J\&W equation (6) for the oil T9 and that the shear stress is limited to $\Lambda p$. The broken curve is the sinh-law (3) with $\tau_{\mathrm{E}}=5(\mathrm{MPa})$

\section{THE DYNAMIC CROSSOVER FOR TWO LUBRICANTS}

At a temperature, $T_{\mathrm{B}}>T_{\mathrm{g}}$, a transition in dynamics during isobaric cooling is often observed, characterized by a crossover from one VTF relation to another [29]. A similar transition has been observed in the isothermal compression of liquids in which there is a crossover from one J\&W relation to another [30]. This change in dynamics is termed the dynamic crossover and the two forms of the Stickel analysis introduced above clearly detect the effect. The mechanism underlying the dynamic crossover is variously described as in reference [31]: a crossover from free (not intermolecularly correlated) diffusion to energy landscape dominated diffusion with motions governed by the barrier heights between local potential energy minima; the percolation of 'liquid-like cells' according to free-volume models, whereby continuity of empty space facilitates motion; the onset of strong intermolecular cooperativity; the (hoppingmediated) dynamic singularity predicted by mode coupling theory; or the emergence of thermal density fluctuations having a length scale larger than the liquid cage structure, enabling 'jumps' over local barriers. Although the language and details of the various models differ, the common theme is that below $T_{\mathrm{B}}$, the dynamics become 'fully cooperative'; that is, structural relaxation and diffusion of a molecule are strongly correlated with the motions of neighbouring molecules. Nevertheless, even above $T_{\mathrm{B}}$ the $T$-dependence does not assume the Arrhenius form, indicating that intermolecular correlations persist. The dynamic crossover is related to the fragility, with more fragile liquids exhibiting larger changes in dynamics at $T_{\mathrm{B}}[\mathbf{3 2}, \mathbf{3 3}]$.
Table 2 Properties of the liquid lubricants displaying a crossover as supplied by the manufacturer

\begin{tabular}{|c|c|c|}
\hline & MCS 418 & MCS 460 \\
\hline$T(\mathrm{~K})$ & \multicolumn{2}{|c|}{ Kinematic viscosity $\left(\mathrm{mm}^{2} / \mathrm{s}\right)$} \\
\hline 255 & 13000 & 26000 \\
\hline 298 & - & 86.0 \\
\hline 311 & 25 & 37.2 \\
\hline 372 & 4.1 & 4.0 \\
\hline 422 & 2.0 & 1.9 \\
\hline 477 & \multicolumn{2}{|c|}{ Density (g/mL) } \\
\hline 255 & - & 0.9595 \\
\hline 298 & 1.195 & 0.9327 \\
\hline 311 & 1.184 & 0.9219 \\
\hline 372 & - & 0.8822 \\
\hline 422 & 1.101 & 0.8504 \\
\hline 477 & - & 0.8148 \\
\hline
\end{tabular}

Two liquid lubricants discovered to display a dynamic crossover are MCS 418 and MCS 460. These are the model lubricants described by Hamrock et al. [34] as c-ether and synthetic hydrocarbon, respectively. MCS 418 is a polyphenyl thioether [35] used for high temperature aircraft turbine lubrication, having improved low temperature properties over the unsubstituted polyphenyl ethers. MCS 460 is a synthetic cycloaliphatic hydrocarbon traction fluid for continuously variable transmissions. Some properties obtained from the manufacturer (Monsanto) are given in Table 2.

The pressure-volume-temperature (PVT) response was measured in a commercial metal bellows piezometer to pressures up to $200 \mathrm{MPa}$. These data were fitted to the Tait equation of state

$$
\frac{V}{V_{0}(T)}=1-\frac{1}{1+K_{0}^{\prime}} \ln \left[1+\frac{p}{K_{0}(T)}\left(1+K_{0}^{\prime}\right)\right]
$$

The specific volume at ambient pressure is $V_{0}(T)=$ $a_{0}+a_{1} T+a_{2} T^{2}$ and the bulk modulus at ambient pressure is $K_{0}=K_{00} \exp \left(-\beta_{K} T\right)$. Parameters for the equation of state are listed in Table 3.

The viscosities of the two liquids were measured in falling body viscometers [36]. One of these viscometers

Table 3 Parameters of the equation of state for the liquid lubricants displaying a crossover

\begin{tabular}{lll}
\hline & MCS 418 & MCS 460 \\
\hline$K_{0}^{\prime}$ & 10.574 & 10.574 \\
$K_{00}(\mathrm{GPa})$ & 9.69 & 9.45 \\
$\beta_{\mathrm{K}}\left(\mathrm{K}^{-1}\right)$ & 0.004438 & 0.005007 \\
$a_{0}\left(\mathrm{~m}^{3} \mathrm{~kg}^{-1}\right)$ & $0.82367 \times 10^{-3}$ & $1.05389 \times 10^{-3}$ \\
$a_{1}\left(\mathrm{~m}^{3} \mathrm{~kg}^{-1} \mathrm{~K}^{-1}\right)$ & $5.1839 \times 10^{-7}$ & $6.901 \times 10^{-7}$ \\
$a_{2}\left(\mathrm{~m}^{3} \mathrm{~kg}^{-1} \mathrm{~K}^{-2}\right)$ & $2.187 \times 10^{-10}$ & $3.2335 \times 10^{-10}$ \\
\hline
\end{tabular}


Table 4 The viscosity of MCS 418 in Pa s

\begin{tabular}{clll}
\hline$p$ (MPa) & $293 \mathrm{~K}$ & $323 \mathrm{~K}$ & $373 \mathrm{~K}$ \\
\hline 0 & 0.0819 & & \\
50 & 0.268 & & \\
100 & 1.103 & & \\
150 & 6.33 & 0.204 & \\
200 & 56 & 0.567 & \\
250 & 808 & 1.841 & \\
300 & 17400 & 8.01 & \\
330 & 135000 & & \\
350 & & 44.6 & 0.195 \\
400 & & 361 & 0.395 \\
450 & & 3590 & 0.883 \\
500 & & 59700 & 2.15 \\
550 & & & 6.01 \\
600 & & & 18.5 \\
650 & & & 71.8 \\
700 & & & 361 \\
750 & & & 1984 \\
800 & & & 13700 \\
850 & & & 141000 \\
\hline
\end{tabular}

was modified for this purpose to improve the temperature control for conditions which combine high temperature with large viscosity. Temperature of the pressure vessel is controlled by the flow of heated air through passages in the vessel wall. Feedback control was added to maintain within $\pm 0.2 \mathrm{~K}$ the temperature of the air at the inlet to the vessel. Viscosities are listed in Tables 4 and 5 for MCS 418 and MCS 460, respectively.

The conventional presentation of these data as the logarithm of viscosity versus pressure is shown in Fig. 5 for the polyphenyl thioether. The appearance of the isotherms is normal with faster than exponential pressure response apparent at high pressure. The pressure-Stickel plot of Fig. 6 indicates a transition in the pressure-viscosity response from a low pressure

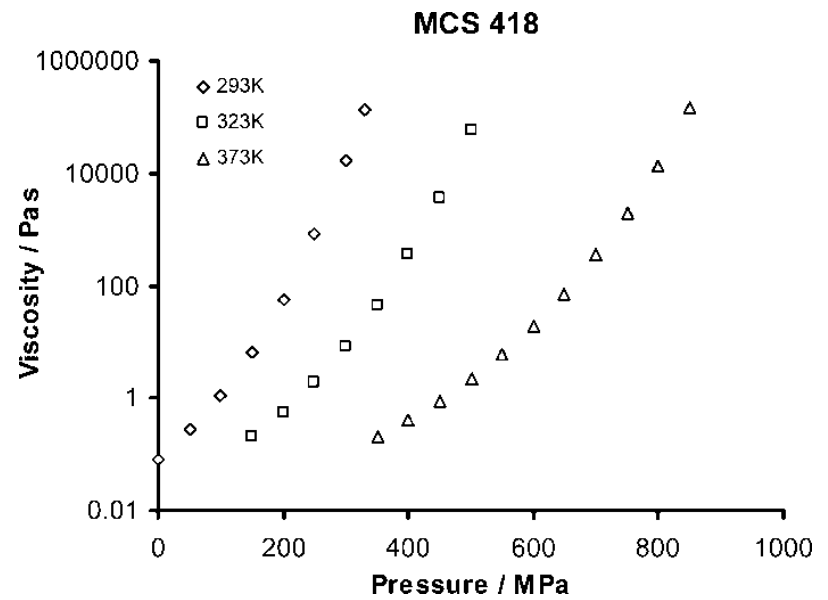

Fig. 5 The logarithm of viscosity of polyphenyl thioether versus pressure

$\mathrm{J} \& \mathrm{~W}$ equation to another at high pressure having a less rapid decrease in $\phi_{\mathrm{p}}$ with pressure. This change in response corresponds to a less rapid increase in $\alpha$ with pressure at high pressure as compared to low pressure. The parameters of the J\&W equation are listed in Table 6.

The viscosity at the dynamic crossover is plotted versus reciprocal temperature in Fig. 7. In comparison with the temperature dependence of viscosity at $p=350 \mathrm{MPa}$, shown as the triangles in Fig. 7, the crossover viscosity is reasonably constant at $\sim 90 \mathrm{~Pa}$ s for MCS 418. The glass transition pressure, measured by isothermal compression in a dilatometer [37], is plotted in Fig. 8. The temperature dependence of the crossover pressure is the same as the temperature dependence of the glass transition pressure. This is not surprising since the glass transition corresponds to an isoviscous state.

Table 5 The viscosity of MCS 460 in Pa s

\begin{tabular}{|c|c|c|c|c|c|c|c|c|c|c|c|c|c|c|}
\hline$p(\mathrm{MPa})$ & $293 \mathrm{~K}$ & $313 \mathrm{~K}$ & $338 \mathrm{~K}$ & $373 \mathrm{~K}$ & $388 \mathrm{~K}$ & $308.3 \mathrm{~K}$ & $317.1 \mathrm{~K}$ & $326 \mathrm{~K}$ & $334.1 \mathrm{~K}$ & $345.6 \mathrm{~K}$ & $356.2 \mathrm{~K}$ & $369.1 \mathrm{~K}$ & $383.9 \mathrm{~K}$ & $400 \mathrm{~K}$ \\
\hline 0 & & & & & 0.00277 & & & & & & & & & \\
\hline 25 & 0.326 & & & & & & & & & & & & & \\
\hline 50 & 1.072 & 0.143 & & & 0.00626 & & & & & & & & & \\
\hline 75 & & 0.371 & & & & & & & & & & & & \\
\hline 100 & 15.2 & 1.01 & 0.118 & & 0.0142 & & & & & & & & & \\
\hline 125 & 67.7 & 2.87 & & & & & & & & & & & & \\
\hline 150 & 336 & 8.76 & 0.569 & & 0.0335 & & & & & & & & & \\
\hline 175 & 1831 & 30.1 & & & & & & & & & & & & \\
\hline 200 & 9532 & 110.9 & 3.21 & 0.18 & 0.0846 & & & & & & & & & \\
\hline 225 & 49140 & 450 & & & & & & & & & & & & \\
\hline 250 & & 1794 & 23.8 & 0.631 & 0.235 & & & & & & & & & \\
\hline 275 & & 6960 & & & & & & & & & & & & \\
\hline 300 & & 28200 & 215 & 2.56 & 0.731 & 79270 & 11000 & 1767 & 414 & 61.9 & 15.6 & 3.69 & 0.981 & 0.306 \\
\hline 350 & & & 1740 & 11.4 & 2.52 & & & & & & & & & \\
\hline 400 & & & 15500 & 53.7 & 10.28 & & & & & & & & & \\
\hline 450 & & & & 330 & 40 & & & & & & & & & \\
\hline 500 & & & & 1540 & 202 & & & & & & & & & \\
\hline 550 & & & & 7840 & 776 & & & & & & & & & \\
\hline 600 & & & & & 4131 & & & & & & & & & \\
\hline
\end{tabular}




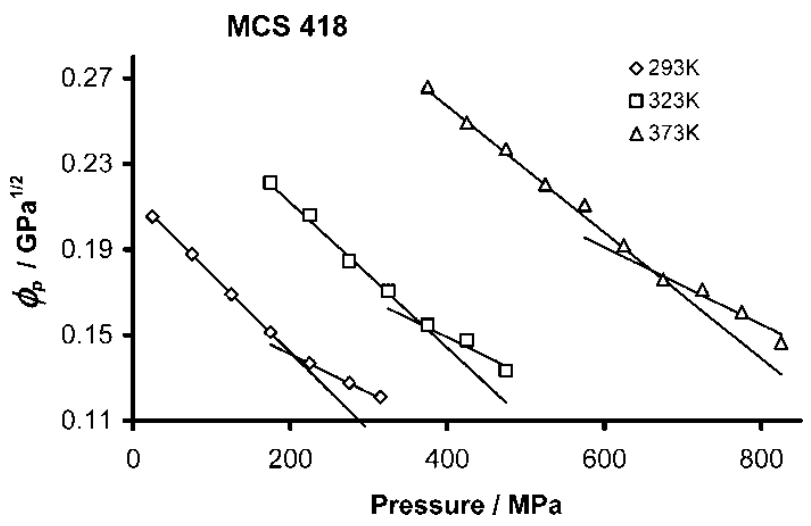

Fig. 6 The pressure-Stickel plot for the polyphenyl thioether showing the crossover from one $\mathrm{J} \& \mathrm{~W}$ relation at low pressure to another at high pressure

Table 6 Parameters of the J\&W equation for the low pressure and high pressure regimes of the polyphenyl thioether, MCS 418

\begin{tabular}{lccc}
\hline & $293 \mathrm{~K}$ & $323 \mathrm{~K}$ & $373 \mathrm{~K}$ \\
\hline Low pressure regime & & & \\
$C_{\mathrm{F}}$ & 12.74 & 10.50 & 9.04 \\
$p_{\infty}(\mathrm{GPa})$ & 0.589 & 0.824 & 1.27 \\
High pressure regime & & & \\
$C_{\mathrm{F}}$ & 31.4 & 25.4 & 18.6 \\
$p_{\infty}(\mathrm{GPa})$ & 0.983 & 1.23 & 1.66 \\
\hline
\end{tabular}

The pressure-viscosity isotherms for the cyloaliphatic hydrocarbon, MCS 460, are shown in the Stickel plot of Fig. 9. Again, there is a crossover from one J\&W relation to another at a characteristic pressure for each temperature. The parameters of the J\&W equation are listed in Table 7 . For the high pressure regime, $\phi_{\mathrm{p}}$, is reasonably

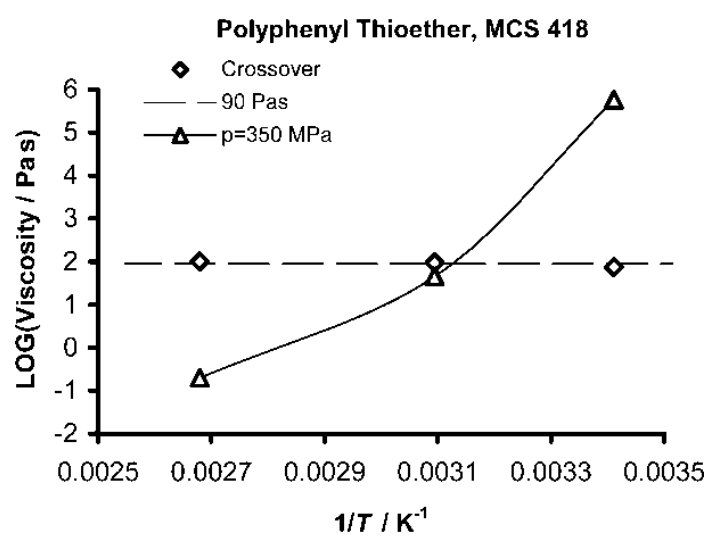

Fig. 7 The viscosity of polyphenyl thioether at the dynamic crossover compared with the viscosity at a constant pressure of $350 \mathrm{MPa}$. The viscosity at the crossover is approximately constant with a value $\sim 90 \mathrm{Pas}$

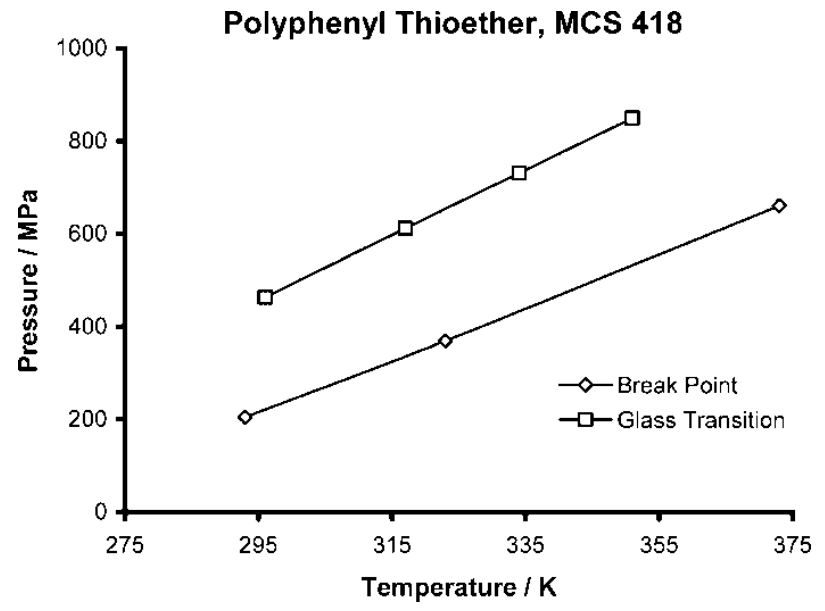

Fig. 8 The glass transition pressure by isothermal compression compared with the pressure at the dynamic crossover

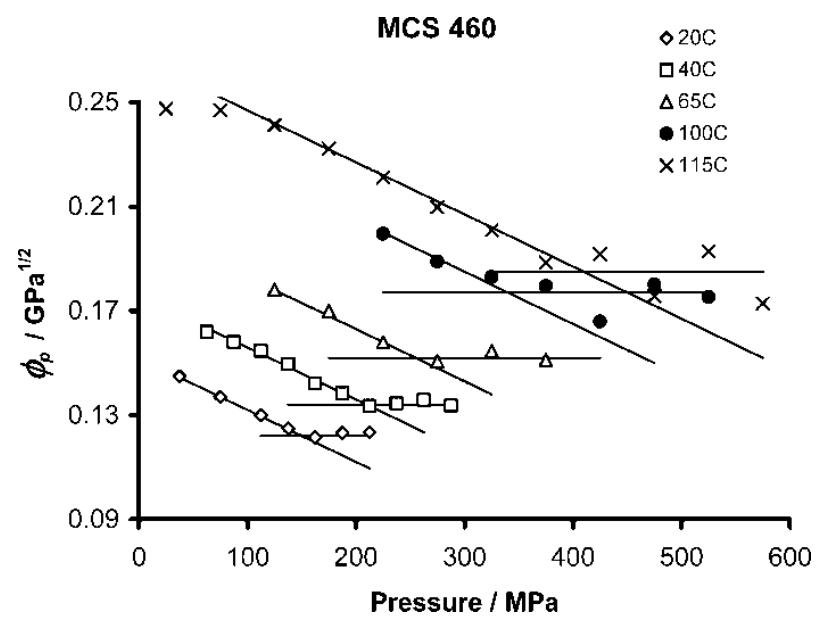

Fig. 9 The pressure-Stickel plot for the cylcoaliphatic hydrocarbon showing the crossover from one $\mathrm{J} \& \mathrm{~W}$ relation at low pressure to another at high pressure

independent of pressure leading to unbounded values for $C_{\mathrm{F}}$ and $p_{\infty}$. Here, $C_{\mathrm{F}} / p_{\infty}=\alpha$ is listed in Table 7 and the exponential law applies.

The isobaric derivative behaviour is similar as shown in Fig. 10 for a pressure of $300 \mathrm{MPa}$. On the temperature-Stickel plot, there is a crossover from one

Table 7 Parameters of the J\&W equation for the low pressure and high pressure regimes of the cylcoaliphatic hydrocarbon, MCS 460

\begin{tabular}{lccccc}
\hline & $293 \mathrm{~K}$ & $313 \mathrm{~K}$ & $338 \mathrm{~K}$ & $373 \mathrm{~K}$ & $388 \mathrm{~K}$ \\
\hline Low pressure regime & & & & & \\
$C_{\mathrm{F}}(\mathrm{GPa})$ & 32.9 & 28.41 & 24.63 & 20.41 & 18.73 \\
$p_{\infty}(0.760$ & 0.880 & 1.015 & 1.225 & 1.335 \\
High pressure regime & & & & & \\
$\quad C_{\mathrm{F}}=\alpha\left(\mathrm{GPa}^{-1}\right)$ & 67.2 & 55.7 & 43.3 & 33.0 & 29.2 \\
\hline$p_{\infty}$ & & & & & \\
\hline
\end{tabular}




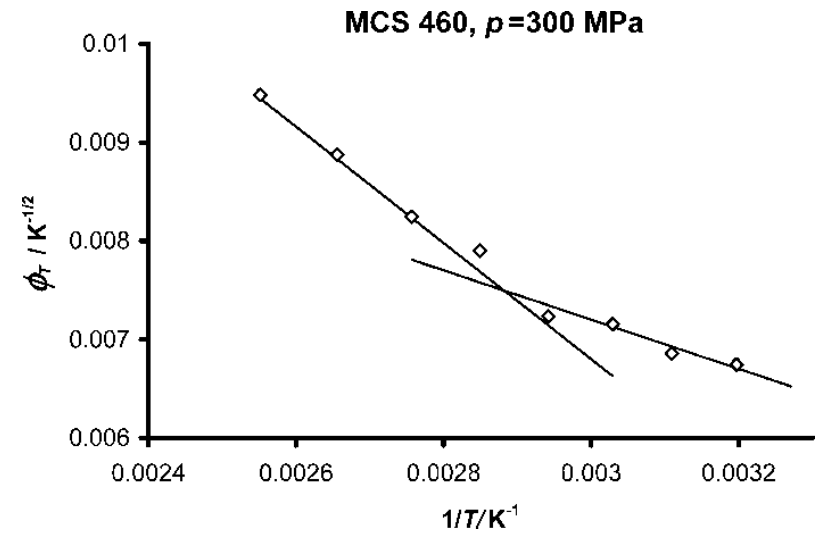

Fig. 10 The temperature-Stickel plot for the cylcoaliphatic hydrocarbon at $300 \mathrm{MPa}$ showing the crossover from one VFT relation at high temperature to another at low temperature

VTF relation (5) at high temperature to another at low temperature in Fig. 10. The VTF parameters are $D_{\mathrm{F}}=6.92$ and 27.2 , and $T_{\infty}=241$ and $170 \mathrm{~K}$ for the high and low temperature regimes respectively.

Crossover viscosities for MCS 460 are plotted against reciprocal temperature in Fig. 11. In comparison with the isobaric temperature dependence at $200 \mathrm{MPa}$, shown as the triangles in the figure, the crossover viscosity is only weakly dependent on temperature, increasing from about 10 to $300 \mathrm{~Pa}$ as the temperature decreases from 388 to $293 \mathrm{~K}$. The glass transition pressure has been measured by isothermal compression and isobaric cooling in dilatometers and plotted in Fig. 12. The dependence of the crossover pressure on

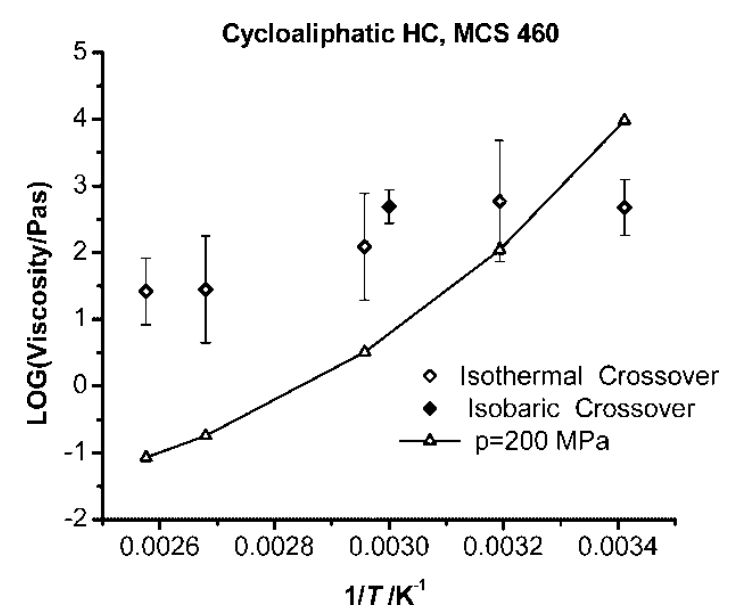

Fig. 11 The viscosity at the dynamic crossover increases slowly with reciprocal temperature for the cylcoaliphatic hydrocarbon in comparison with the temperature dependence of viscosity at $200 \mathrm{MPa}$

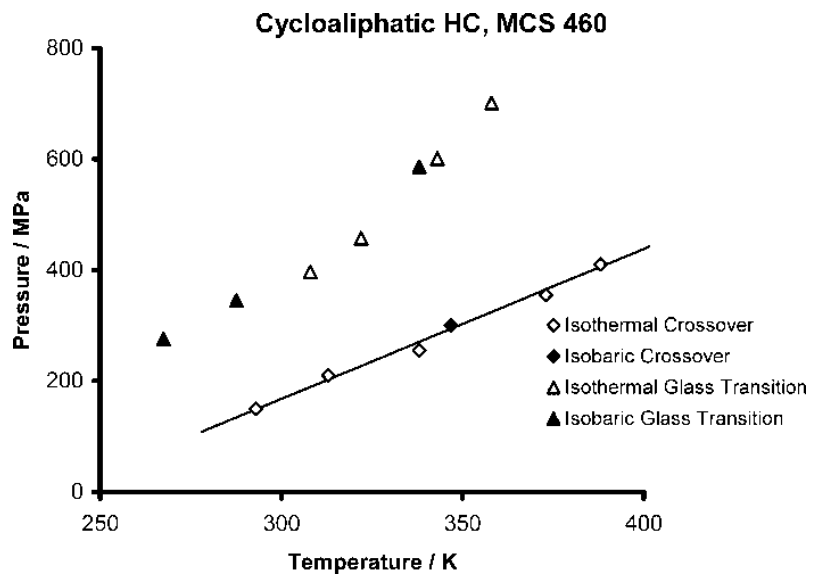

Fig. 12 The glass transition pressure by compression and cooling compared with the crossover pressure by compression and cooling

temperature is slightly weaker than the dependence of the glass transition pressure on temperature.

\section{THE FREE-VOLUME MODEL AND THE CROSSOVER}

The free-volume model has been applied with success to describe the temperature and pressure dependence of the viscosity of lubricants [21], although its general application to the dynamics of supercooled liquids is problematic [31, 38, 39]. Notwithstanding the correctness of the physical interpretation underlying the model, it reproduces the observed trends and yields viscosity values of sufficient accuracy for tribological calculations, if less accurate than experimental determinations. The Doolittle equation

$$
\begin{aligned}
\mu= & \mu_{\mathrm{R}} \exp \left[\frac { B V _ { \infty \mathrm { R } } } { V _ { \mathrm { R } } } \left(\frac{V_{\infty} / V_{\infty \mathrm{R}}}{\left(V / V_{\mathrm{R}}\right)-\left(V_{\infty \mathrm{R}} / V_{\mathrm{R}}\right)\left(V_{\infty} / V_{\infty \mathrm{R}}\right)}\right.\right. \\
& \left.\left.-\frac{1}{1-\left(V_{\infty \mathrm{R}} / V_{\mathrm{R}}\right)}\right)\right]
\end{aligned}
$$

relates viscosity to the total volume, $V$, and the occupied volume, $V_{\infty}$. The subscript, 'R' refers to a reference state, $T_{\mathrm{R}}=293 \mathrm{~K}$ and $p=0$, and it is assumed that the occupied volume depends only on temperature as

$$
\frac{V_{\infty}}{V_{\infty \mathrm{R}}}=1+\varepsilon\left(T-T_{\mathrm{R}}\right)
$$

Employing the Tait equation of state (14) for the volume, the free-volume parameters were regressed from the viscosity data for MCS 460 at viscosities less than the value at the crossover. The regressed values are $\mu_{\mathrm{R}}=0.1551 \mathrm{~Pa} \mathrm{~s}, B=0.8754, V_{\infty \mathrm{R}} / V_{\mathrm{R}}=0.8987$, and $\varepsilon=-3.801 \times 10^{-4} \mathrm{~K}^{-1}$ with standard deviation of 


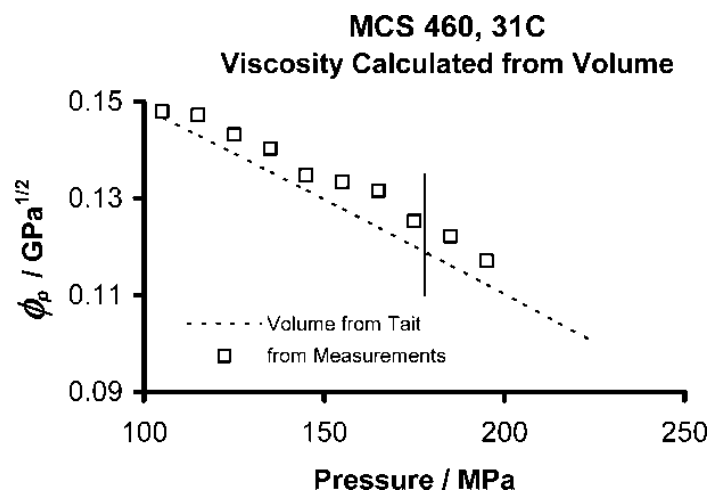

Fig. 13 The free-volume model was fitted to viscosity data below the crossover. This pressure-Stickel plot was created from the free-volume viscosity obtained from volumes calculated from the Tait equation of state (EOS) and from measured volumes. The interpolated crossover pressure is indicated by the vertical line

9 per cent. Then the model was used to construct the pressure-Stickel plot in Fig. 13. Here both the Tait equation and the measured values for the volume of MCS 460 were utilized in the viscosity calculation. First, notice that the linear descending regime of the Stickel analysis that is described by the J\&W equation is reproduced well by the free-volume model with the Tait equation. The temperature for this exercise, $304 \mathrm{~K}$, was chosen because there are directly measured volumes available at this temperature in an interval of pressure that includes the crossover. The linear curve through the crossover data in Fig. 12 extrapolates to a pressure of $178 \mathrm{MPa}$ at $304 \mathrm{~K}$. This pressure is marked as the vertical line in Fig. 13. The free-volume relation has been the only model used in EHL simulations to accurately describe fragility. The $\phi_{\mathrm{p}}(p)$ obtained from the free-volume model does not display the crossover; clearly, the free-volume model cannot reproduce the dynamic crossover.

\section{CONCLUSION}

1. The pressure fragility property is important to EHL traction and appears to be the controlling parameter for the representative (Eyring) stress.

2. The Stickel derivative analysis may be applied to lubricants to detect the dynamic crossover in either the temperature-viscosity or the pressure-viscosity response.

3. The viscosity at the crossover is either constant or varies slowly with temperature and pressure.

4. The free-volume model fails to predict the dynamic crossover when it occurs and a modification of the viscosity law should be considered when the crossover is known to occur.

\section{REFERENCES}

1 Liu, Y., Wang, Q. J., Wang, W., Hu, Y., Zhu, D., Krupka, I., and Hartl, M. EHL simulation using the free-volume viscosity model. Tribol. Lett., 2006, 23(1), 27-37.

2 Reynolds, 0 . On the theory of lubrication and its application to Mr Beauchamp tower's experiments including an experimental determination of the viscosity of olive oil. Philos. Trans. R. Soc. Lond., 1886, 177, 157-234.

3 Dowson, D. A generalized Reynolds equation for fluid film lubrication. Int. J. Mech. Sci., 1962, 4, 159-170.

4 Yang, P., Kaneta, M., and Masuda, S. Closure to discussion: 'quantitative comparisons between measured and solved dimples in point contacts.' (Yang, P., Kaneta, M., and Masuda, S. ASME J. Tribol., 2003, 125(1), 210-214), ASME J. Tribol., 2005, 127, 457.

5 Brix, V. H. Discussion of 'Hydrodynamic theory in gear lubrication'. (Cameron, A. J. Inst. Pet., 1952, 38(345), 614-623).

6 Bair, S., Khonsari, M., and Winer, W. O. High-pressure rheology of lubricants and limitations of the Reynolds equation. Tribol. Int., 1998, 31(10), 573-586.

7 Rajagopal, K. R. and Szeri, A. Z. On an inconsistency in the derivation of the equations of elastohydrodynamic lubrication. Proc. R. Soc. Lond. A, 2003, 459, 2771-2786.

8 Hirst, W. and Moore, A. J. The effect of temperature on traction in elastohydrodynamic lubrication. Philos. Trans. R. Soc Lond. A, 1980, 298(1438), 183-208.

9 Johnson, K. L. and Tevaarwerk, J. L. Shear behaviour of elastohydrodynamic oil films. Proc. R. Soc. Lond. A, 1977, 356, 215-236.

10 Habchi, W., Demirci, I., Eyheramendy, D., MoralesEspejel, G., and Vergne, P. A finite element approach of thin film lubrication in circular EHD contacts. In Tribology International, 33rd Leeds-Lyon Symposium on Tribology Special Issue, Leeds, UK, 2007, paper TRIBINTD-06-00173R1.

11 Liu, Y., Wang, J. Q., Bair, S., and Vergne, P. A quantitative solution for the full shear-thinning EHL point contact problem including traction. In Proceedings of the Joint Tribology Conference IJTC2007, 2007, paper 44100.

12 Oldekop, V. W. Theoretische betrachtungen über die Zähigkeit von Gläsern. Glastechnisch Berichte, 1957, 30, 8-14.

13 Laughlin, W. T. and Uhlmann, D. R. Viscous flow in simple organic liquids. J. Phys. Chem., 1972, 76, 2317-2325.

14 Angell, C. A. Relaxation in liquids, polymers and plastic crystals-strong/fragile patterns and problems. J. NonCryst. Solids, 1991, 131-133, 13-31.

15 Angell, C. A. Formation of glasses from liquids and biopolymers. Science, 1995, 276, 1924-1935.

16 Andrade, E. N. da C. The viscosity of liquids. Nature, 1930, 125, 309-310.

17 Johari, G. P. and Whalley, E. Dielectric properties of glycerol in the range $0.1-10^{5} \mathrm{~Hz}, 218-357 \mathrm{~K}, 0-53 \mathrm{~kb}$. Faraday Symp. Chem. Soc., 1972, 6(6), 23-41.

18 Bohmer, R., Ngai, K. L., Angell, C. A., and Plazek, D. J. Non-exponential relaxations in strong and fragile glass formers. J. Chem. Phys., 1993, 99(5), 4201-4209.

19 Paluch, M., Gapinski, J., Patkowski, A., and Fischer, E. W. Does fragility depend on pressure? A 
dynamic light scattering study of a fragile glass former. J. Chem. Phys., 2001, 114(18), 8048-8055.

20 Paluch, M., Denzik, Z., and Rzoska, S. J. Scaling of high-pressure viscosity data in low-molecularweight glass-forming liquids. Phys. Rev. B, 1999, 60(5), 2979-2982.

21 Bair, S. and Kottke, P. Pressure-viscosity relationships for elastohydrodynamics. STLE Tribol. Trans., 2003, 46, 289-295.

22 Stickel, F., Fischer, E. W., and Richert, R. Dynamics of glass-forming liquids. II. detailed comparison of dielectric relaxation, DC-conductivity, and viscosity data. J. Chem. Phys., 1996, 104(5), 2043-2055.

23 Casalini, R. and Roland, C. M. Viscosity at the dynamic crossover in o-terphenyl and salol under pressure. Phys. Rev. Lett., 2004, 92(24), 245702-1-4.

24 Barus, C. Isothermals, isopiestics and isometrics relative to viscosity. Am. J. Sci. Third Ser., 1893, 16(266), 87-96.

25 Roelands, C. J. A. Correlational aspects of the viscositytemperature-pressure relationship of lubricating oils. PhD Thesis, University of Technology, Delft, 1966, p. 95, 105, 108.

26 Bair, S. Discussion of Greenwood, J., 'Non-Newtonian lubrication'. In Tribology research: from model experiment to industrial problem (Eds G. Dalmaz, A. A. Lubrecht, D. Dowson, and M. Priest), 2001, pp. 945-947 (Elsevier, Amsterdam).

27 Johnson, K. L. and Tevaarwerk, J. L. Shear behaviour of elastohydrodynamic oil films. Proc. R. Soc. Lond. A, 1977, 356, 215-236.

28 Bair, S. and McCabe, C. A study of mechanical shear bands in liquids at high pressure. Tribol. Int., 2004, 37, 783-789.

29 Hansen, C., Stickel, F., Berger, T., Richert, R., and Fischer, E. W. Dynamics of glass-forming liquids. III. Comparing the dielectric a- and b-relaxation of 1propanol and o-terphenyl. J. Chem. Phys., 1997, 104(4) 1086-1093.

30 Casalini, R. and Roland, M. C. Viscosity in o-terphenyl and salol under high pressure. Phys. Rev. Lett., 2004, 92(24), 245702-1-25702-4.

31 Roland, C. M., Hensel-Bielowka, S., Paluch, M., and Casalini, R. Supercooled dynamics of glass-forming liquids and polymers under hydrostatic pressure. Rep. Prog. Phys. 2005, 68, 1405-1478.

32 Leon, C. and Ngai, K. L. Rapidity of the change of the kohlrausch exponent of the $\alpha$-relaxation of glass-forming liquids at $\mathrm{T}_{\mathrm{B}}$ or $\mathrm{T}_{\beta}$ and consequences. J. Phys. Chem. B, 1999, 103, 4045-4051.

33 Novikov, V. N. and Sokolov, A. P. Poisson's ratio and the fragility of glass-forming liquids. Nature, 2004, 43, 961-962.

34 Hamrock, B. J., Schmid, S. R., and Jacobson, B. O. Fundamentals of fluid film lubrication, 2nd edition, 2004, pp. 91-95 (Marcel Dekker, New York).

35 Sulton, D. L., Boothe, M., Ball, D. W., and Morales, W. Electron affinity calculations for thioethers. NASA Technical Memorandum 113178, 1997.

36 Bair, S. A routine high-pressure viscometer for accurate measurements to 1 GPa. STLE Tribol. Trans., 2004, 47(3), 356-360.
37 Bair, S. High shear stress rheology of liquid lubricants. $\mathrm{PhD}$ Thesis, Georgia Institute of Technology, University Microfilms, Ann Arbor, 1990, pp. 40-52.

38 Paluch, M., Casalini, R., and Roland, C. M. Cohen Grest model for the dynamics of supercooled liquids. Phys. Rev. E, 2003, 67, 021508.

39 Floudas, G. In Broadband dielectric spectroscopy (Eds F. Kremer and A. Schonhals), 2003, ch. 8 (Springer-Verlag, Berlin).

\section{APPENDIX}

\section{Notation}

$a_{i} \quad$ parameters for the ambient pressure specific volume $\left(\mathrm{m}^{3} / \mathrm{kg} / \mathrm{K}^{-1}\right)$

$B \quad$ Doolittle parameter

$C_{\mathrm{F}} \quad$ fragility parameter in the Johari and Whalley equation

$C_{\mathrm{E}} \quad$ parameter multiplying $\Sigma$ in the traction sinh law

$D_{\mathrm{F}} \quad$ fragility parameter in the VTF equation

$E_{\mathrm{a}} \quad$ thermal activation energy $(\mathrm{J} / \mathrm{kmol})$

$h \quad$ film thickness (m)

$K_{0} \quad$ isothermal bulk modulus at $p=0(\mathrm{~Pa})$

$K_{0}^{\prime} \quad$ pressure rate of change of isothermal bulk modulus at $p=0$

$K_{00} \quad K_{0}$ at zero absolute temperature (Pa)

$m_{\mathrm{T}} \quad$ temperature fragility index

$m_{\mathrm{p}} \quad$ pressure fragility index $\left(\mathrm{Pa}^{-1}\right)$

$p \quad$ pressure $(\mathrm{Pa})$

$\bar{p} \quad$ average contact pressure $(\mathrm{Pa})$

$p_{\infty} \quad$ pressure at which viscosity diverges $(\mathrm{Pa})$

$p_{\mathrm{g}} \quad$ glass transition pressure $(\mathrm{Pa})$

$p_{\mathrm{H}} \quad$ Hertz (maximum contact) pressure (Pa)

$r$ dimensionless Hertz contact radius

$R_{\mathrm{g}} \quad$ universal gas constant $=8314.34$

$\left(\mathrm{Pa} \mathrm{m}^{3} / \mathrm{kmol} / \mathrm{K}\right)$

$T \quad$ temperature (K)

$T_{\mathrm{B}} \quad$ crossover temperature (K)

$T_{\mathrm{g}} \quad$ glass transition temperature (K)

$T_{\mathrm{R}} \quad$ reference temperature (K)

$T_{\infty} \quad$ divergence temperature $(\mathrm{K})$

$V \quad$ specific volume at $T$ and $p\left(\mathrm{~m}^{3} / \mathrm{kg}\right)$

$V_{\mathrm{R}} \quad$ specific volume at reference state, $T_{\mathrm{R}}$, $p=0\left(\mathrm{~m}^{3} / \mathrm{kg}\right)$

$V_{0} \quad$ specific volume at $p=0\left(\mathrm{~m}^{3} / \mathrm{kg}\right)$

$V_{\infty} \quad$ occupied volume $\left(\mathrm{m}^{3} / \mathrm{kg}\right)$

$V_{\infty \mathrm{R}} \quad$ occupied volume at reference state, $T_{\mathrm{R}}$, $p=0\left(\mathrm{~m}^{3} / \mathrm{kg}\right)$

$z \quad$ Roelands parameter

$\alpha \quad$ local pressure-viscosity coefficient $\left(\mathrm{Pa}^{-1}\right)$

$\alpha^{*} \quad$ reciprocal asymptotic isoviscous pressure coefficient $\left(\mathrm{Pa}^{-1}\right)$ 


$\begin{array}{lll}\alpha_{\mathrm{g}} & \begin{array}{l}\text { pressure-viscosity coefficient of the liquid } \\ \text { at the glass transition }\left(\mathrm{Pa}^{-1}\right)\end{array} & \rho \\ \beta & \text { temperature-viscosity coefficient }\left(\mathrm{K}^{-1}\right) & \rho_{\mathrm{R}} \\ \beta_{\mathrm{K}} & \text { temperature coefficient of } K_{0}\left(\mathrm{~K}^{-1}\right) & \Sigma \\ \dot{\gamma} & \text { shear rate }\left(\mathrm{s}^{-1}\right) & \\ \eta & \text { rate-dependent shear viscosity (Pa s) } & \tau \\ \Lambda & \text { limiting stress-pressure coefficient } \\ \mu & \text { limiting low-shear viscosity (Pa s) } & \tau_{\mathrm{a}} \\ \mu_{0} & \text { low-shear viscosity at } p=0 \text { (Pa s) } \\ \mu_{\mathrm{g}} & \text { low-shear viscosity at the glass transition } \\ & \begin{array}{l}\text { (Pas) } \\ \mu_{\mathrm{R}}\end{array} & \text { low-shear viscosity at reference state, } \\ & T_{\mathrm{R}}, p=0 \text { (Pa s) } \\ \mu_{\infty} & \text { low-shear viscosity at infinite } T \text { or } p \text { (Pas) } & \phi_{\mathrm{T}} \\ \end{array}$

mass density $\left(\mathrm{kg} / \mathrm{m}^{3}\right)$

mass density, at reference state, $T_{\mathrm{R}}, p=0$ $\left(\mathrm{kg} / \mathrm{m}^{3}\right)$

$\Sigma \quad$ sliding/rolling velocity or slide-to-roll ratio

$\tau \quad$ shear stress (Pa)

$\tau_{\text {ave }} \quad$ shear stress averaged over the contact area $(\mathrm{Pa})$

$\tau_{\mathrm{E}} \quad$ representative shear stress, the rate of change of the average shear stress with respect to the natural logarithm of the sliding velocity $(\mathrm{Pa})$

$\phi_{\mathrm{T}} \quad$ the temperature-Stickel function $\left(\mathrm{K}^{1 / 2}\right)$

$\phi_{\mathrm{p}}$ the pressure-Stickel function $\left(\mathrm{Pa}^{-1 / 2}\right)$ 